\title{
The Echo Modelling and Simulation of the Semi-Active Radar Seeker against a Sea Skimming Target
}

\author{
Peng Peng, Lixin Guo, Hualong Sun \\ School of Physics and Optoelectronic Engineering, Xidian University, Xi'an, China \\ Email: pengpengxidian@163.com
}

How to cite this paper: Peng, P., Guo, L.X. and Sun, H.L. (2018) The Echo Modelling and Simulation of the Semi-Active Radar Seeker against a Sea Skimming Target. Journal of Computer and Communications, 6, 74-79.

https://doi.org/10.4236/jcc.2018.612007

Received: September 15, 2018 Accepted: December 23, 2018 Published: December 26, 2018

\begin{abstract}
This paper has proposed a new modelling and simulating technique for the echo of the semi-active radar seeker against the sea skimming target. The echo modelling is based on the electromagnetic scattering mechanisms. A modified Four-path model based on the radar detection scene is used to describe the multipath scattering between the target and rough sea surface. A Facet-based Small Slope Approximation (FBSSA) method is employed to calculate the scattering from the sea surface. The Physical Optics (PO) and the Equivalent Edge Current (EEC) Method is used to calculate the target scattering. In the echo simulations. The results present the original echo and the echo processed by the signal processing procedures, where the clutter and multipath effect can be observed.
\end{abstract}

\section{Keywords}

Semi-Active Radar Seeker, Sea Skimming Target, Electromagnetic Scattering, Radar Echo

\section{Introduction}

Topics about the ocean securities are widely focused in the recent years. The detection and defending against the sea skimming target is one of those topics, and it is particularly noticed in the military field [1] [2]. The semi-active RF seeker is often set up on an airborne or missile borne platform. It doesn't contain an active radar but receives the scattered energy from the target when it's illuminated by the launch platform on the ground. The radar echo components received by the seeker are very complex, where the combined effect of multipath scattering and sea clutter may cause detrimental effects on the target detections. To better 
analyze the echo from the sea skimming target and evaluate the performance of the semi-active seeker detections, an accurate and efficient simulation model is very necessary. An echo simulation model for the semi-active Radar seeker against a sea skimming target is proposed in this paper. The echo simulations are made based on the hybrid electromagnetic scattering mechanisms of the target-sea model, which has considered the scattering in the simulation scene at each time instant.

\section{Approach and Models}

\subsection{The Scattering Mechanisms and Models}

The sea skimming target has composite scattering mechanisms, which basically contain the target scattering, sea clutter scattering and the multipath scattering between the target and sea surface. Four-path model is an efficient way to describe the scattering mechanisms. In this paper, the four path model is employed based on the semi-active seeker detection geometry. The geometry is shown as in the following figure.

As shown in Figure 1, at the receiver, the total response observed can be seen as the summation of the returns from the following paths: the direct return from the target and sea surface; the single bounce return, in which the scattered field experiences a single bounce with the sea surface either after scattering from the target or prior to illuminating the target; The forth path is a double ground bounce return, where the ray interacts with the sea surface twice, once on the path to the target and again after scattering from the target prior to arrival at the receiver. In fact, the classical Four-path model is only suitable in the ideal condition when the sea surface is regarded as a flat surface. The actual sea surface is a kind of highly random rough surface. In this condition, the scattering components are considered at every reflecting facet on the interface boundary, rather than at a singular point as illustrated in Figure 2, which is referred as the modified four-path model or the extended image method [3].

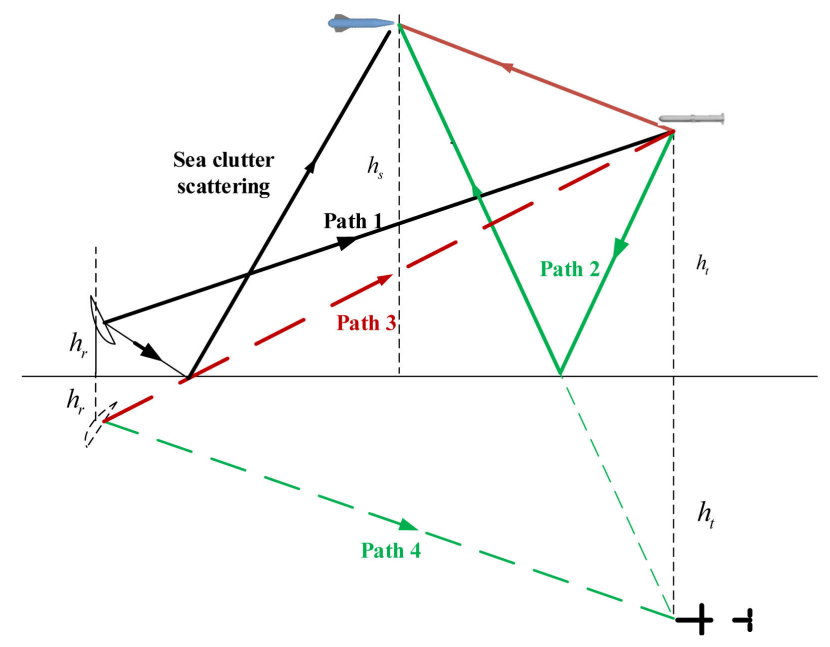

Figure 1. Radar scattering geometry. 


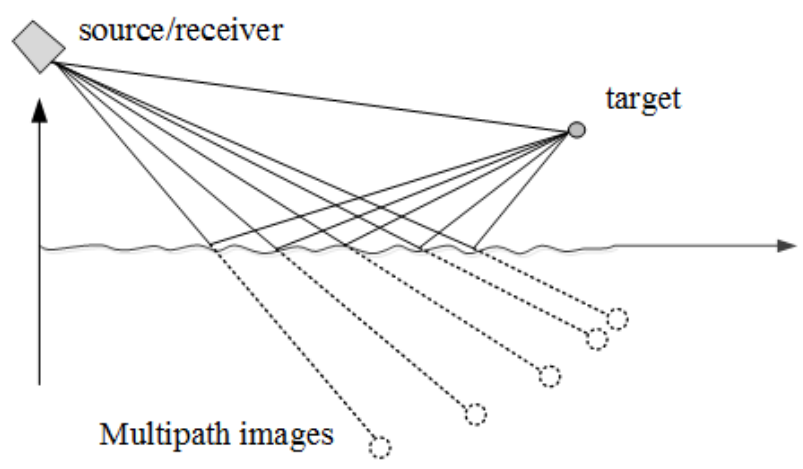

Figure 2. The multipath scattering mechanisms for the very rough surface.

In this model each facet can serve as a small mirror, against which target will yield image reflections at the corresponding image locations. The details of the method can be acquired in the ref. [3]. So in this model, the scattered field upon the illuminated target facet, can be calculated by the Physical Optics method (PO). The scattered field is calculated by the Stratton-Chu equation in PO approximations as given by

$$
\boldsymbol{E}_{s}^{P O}=\frac{j k \mathrm{e}^{-i k R}}{4 \pi R} \iint \eta \cdot \hat{\boldsymbol{k}}_{s} \times\left(\hat{\boldsymbol{k}}_{s} \times\left(2 \boldsymbol{n} \times \boldsymbol{H}_{i n c}\left(\boldsymbol{r}^{\prime}\right)\right)\right) \mathrm{e}^{i k \hat{\boldsymbol{k}}_{s} \cdot \boldsymbol{r}^{\prime}} d s^{\prime}
$$

The Gaussian pattern beam is used as the incident wave. The scattering from the edge of the target is calculated by the Equivalent Edge Current (EEC) method. The scattered field upon the sea facet is calculated by a Facet-based Small Slope Approximation method (FBSSA), where the scattering field upon each sea facet can be calculated by

$$
E_{s}^{S S A}=S^{S S A} \cdot \mathrm{e}^{i k R / R}
$$

$\mathrm{S}^{\mathrm{SSA}}$ is the scattering amplitude (SA) upon an individual sea facet, which is given by

$$
S^{S S A}=\frac{2\left(q q_{0}\right)^{1 / 2}}{\left(q+q_{0}\right) P_{i n c}^{1 / 2}} \boldsymbol{B}\left(\boldsymbol{k}, \boldsymbol{k}_{0}\right) \int \frac{T(\boldsymbol{r}, \xi(\boldsymbol{r}))}{(2 \pi)^{2}} e^{-i\left(\boldsymbol{k}-\boldsymbol{k}_{0}\right) \cdot \boldsymbol{r}-i\left(q+q_{0}\right) \xi(\boldsymbol{r})} d \boldsymbol{r}
$$

$k_{0}$ and $q_{0}$ are the horizontal and vertical projections of the incident vector $k_{i} k$ and $q$ are the horizontal and vertical projections of the scattering vector $k_{s^{*}} B(k$, $k_{0}$ ) is a polarization matrix which can be referred in [4]. The SSA integral can be analytically solved upon the Bragg wave structure on each facet. This process is described in details in the ref. [5], which is not repeated here.

\subsection{Echo Simulation Model}

The echo simulation model is set up in this section for the radar detection scene of the semi-active seeker, which is described in Figure 1. The target is flying above the time-varying sea. The seeker is flying towards the target. The seeker is receiving the scattering return from the target scattering, multipath scattering and sea surface scattering, when they are illuminated by the radar. The radar is assumed to be the pulsed radar. In the pulse duration, the target and the seeker 
are assumed to be static. Considering the fact that the sea surface scattering and the multipath scattering is time varying, so the reflectivity of each scattering point at every position should be calculated at each pulses. The reflectivity of each scattering point is calculated at each time instant. The echo is obtained at each pulse instant by summing up the scattering energy from the scatterers in the simulation scene, which is presented as

$$
s_{r}(k, t)=\sum_{i=1}^{N} \gamma_{i}(k) \cdot \operatorname{rect}\left[\frac{t-2 R_{i}(k) / c}{T_{r}}\right] \varphi\left(t-\frac{2 R_{i}(k)}{c}\right) \exp \left\{-j \frac{4 \pi R_{i}(k)}{\lambda}\right\}
$$

In (4), rect(.) is the envelope of the rectangular pulse. $T_{r}$ is the pulse repetition time (PRT). $R$ is the distance, which can reflect the time delay for the wave propagation. $\gamma_{i}$ is the reflectivity of each scattering point for each time instant, which is calculated by the model in the Section 2.1. The radar scattering returns collected from the facets in the same range bin are processed together.

In the echo simulations, the antenna pattern for the seeker and the ground radar should be considered, where the Gaussian Beam function is used as given by

$$
G_{M B}(\theta)=\exp \left(-2 \ln 2 \theta^{2} / \theta_{B}^{2}\right) \quad|\theta| \leq \mu
$$

$\theta_{B}$ is the half-power beam width of the main lobe. $\mu$ is the azimuth width, given by

$$
\mu=\theta_{B} \sqrt{\ln g_{3} /(-2 \ln 2)}
$$

The radiation from the first and second side-lobes are considered, whose beam function are similarly presented as

$$
\begin{array}{cc}
G_{B 1}(\theta)=g_{1} \exp \left(-2 \ln 2(\theta \pm 1.5 \mu)^{2} / \theta_{B 1}^{2}\right) & \mu \leq|\theta| \leq 2 \mu \\
G_{B 2}(\theta)=g_{2} \exp \left(-2 \ln 2(\theta \pm 2.5 \mu)^{2} / \theta_{B 2}^{2}\right) & 2 \mu<|\theta| \leq 3 \mu
\end{array}
$$

$g_{3}$ is the gain of the main beam. $g_{1}$ is the peak gain of the first side-lobe. $g_{2}$ is the peak gain of the second side-lobe. $\theta_{B 1}$ and $\theta_{B 2}$ are the beam width of the first and second side-lobe, whose value are given by

$$
\begin{aligned}
& \theta_{B 1}=0.5 \mu \sqrt{(-2 \ln 2) / \ln \left(g_{3} / g_{1}\right)} \\
& \theta_{B 2}=0.5 \mu \sqrt{(-2 \ln 2) / \ln \left(g_{3} / g_{2}\right)}
\end{aligned}
$$

In the real scene, the target is flying above the infinite sea surface. The radar echo simulations, the target is moving and the sea surface is time varying. Once the echo is generated, the echo signal is handled by the following signal processing procedures [6] (Figure 3).

\section{Numerical Results}

In the numerical simulations, the working frequency of the ground radar is 10 $\mathrm{GHz}$ (X band). The sea state is chosen as low sea state, where the wind speed at $10 \mathrm{~m}$ high above the sea in the Elfouhaily's sea spectrum is $3 \mathrm{~m} / \mathrm{s}$. 
Figure 4 shows the bistatic scattering characteristics of a cruise missile target above the sea. The target is $5 \mathrm{~m}$ high above the sea, the incident angle is $\theta_{i}=45^{\circ}, \varphi_{i}=0^{\circ}$. The results are compared with the target scattering and the sea scattering. It can be seen that the composite scattering is contributed by the target scattering, sea scattering as well as the multipath scattering, so it's much stronger than each individual scattering component.

In the echo simulations, the bandwidth is $5 \mathrm{MHz}$. The antenna parameters are set as $g_{1}=10^{-2}, g_{2}=10^{-2.5}, g_{3}=10^{-3}$, and $\theta_{B}=5^{\circ}$. The PRT is $10 \mu$ s. The pulse duration is $1 \mu \mathrm{s}$. The sampling frequency is $100 \mathrm{MHz}$ (Figure 5).

Figure 5 shows the echo simulation results, where 512 pulses are used in a CPI. In Figure 5(a), the raw echo signal for. In Figure 5(b), the range-dopper map is obtained by employing the signal processing to the echo. The sea clutter has the strongest power in the map. The target has a velocity, so it has different Doppler value from the clutter. The multipath effect can be also identified in the map, which has broadened the target Doppler spectrum, but it is weak compared with the target and sea clutter.

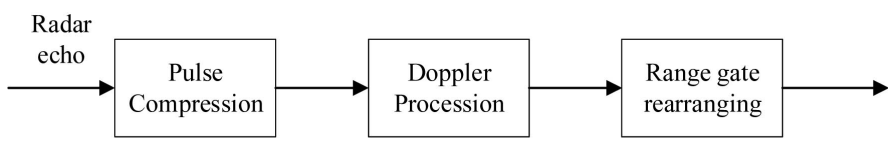

Figure 3. Signal processing procedure.

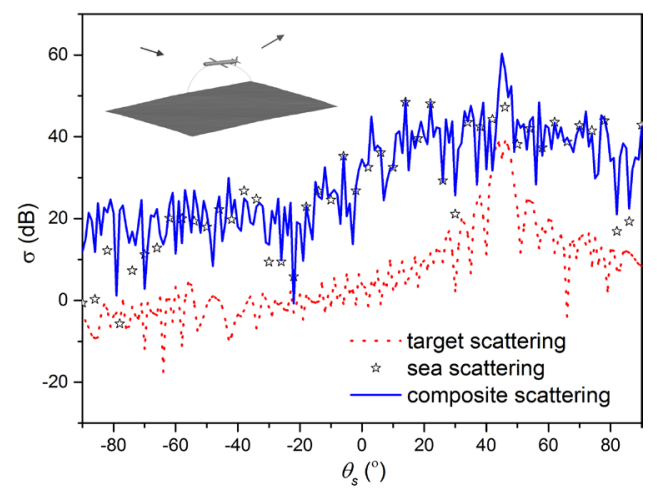

Figure 4. Bistatic scattering characteristics of a cruise missile above the sea surface.

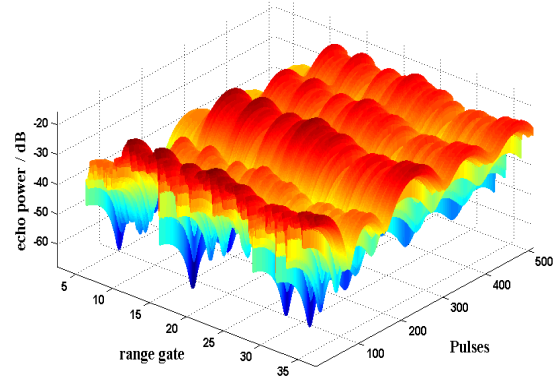

(a)

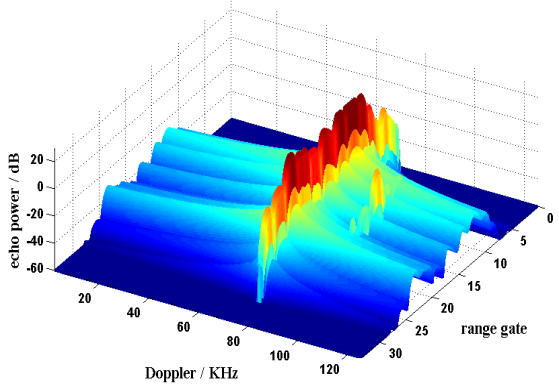

(b)

Figure 5. Echo simulation results of the semi-active seeker. (a) Raw echo signal; (b) Range-Doppler map. 


\section{Conclusion}

In this paper, the radar echo of the semi-active radar seeker against the sea skimming target is modelled and simulated. The scattering mechanisms are considered by a hybrid scheme based on the radar detection scene of the semi-active seeker. The echo after signal processing can show the spatial and Doppler characteristics of the target, sea clutter, and the multipath scattering.

\section{Acknowledgements}

This work was supported by the National Natural Science Foundation of China (Grant No.61431010).

\section{Conflicts of Interest}

The authors declare no conflicts of interest regarding the publication of this paper.

\section{References}

[1] Li, H., Zhang, Y., Li, S., Li, S. and Sun, C. (2010) Low Altitude Sea-Skimming Target Detection System Design of Microwave. Electronic Test, 4, 281-284.

[2] Zhou, H., Guoping, H.U., Kuang, X. and Shi, J. (2017) A Study on the Target Detection Performance of Radar in Low-Altitude Multipath Environment. Modern Radar, 6, 121-124.

[3] Peng, P., Guo, L.X. and Tong, C. (2018) An EM Model for Radar Multipath Simulation and hrrp Analysis of Low Altitude Target above Electrically Large Composite Scale Rough Surface. Electromagnetics, 10, 1-12.

[4] Voronovich, A.G. (2002) The Effect of the Modulation of Bragg Scattering in Small-Slope Approximation. Waves in Random Media, 12, 341-349. https://doi.org/10.1088/0959-7174/12/3/306

[5] Peng, P. and Guo, L. A Facet-Based Simulation of the Multipath Effect on the EM Scattering and Doppler Spectrum of a Low-Flying Target at Maritime Scene. IEEE Geoscience and Remote Sensing Letters.

[6] Melvin, W.L. and Scheer, J.A. (2013) Principles of Modern Radar. An Imprint of the IET, SciTech Publishing, Edison, NJ. 